Noname manuscript No.

(will be inserted by the editor)

Edmund Schluessel

\title{
Constraining the parameters of binary systems through time-dependent light deflection
}

the date of receipt and acceptance should be inserted later

\begin{abstract}
A theory is derived relating the configuration of the cores of active galaxies, specifically candidates for presumed super-massive black hole binaries (SMBHBs), to time-dependent changes in images of those galaxies. Three deflection quantities, resulting from the monopole term, mass quadrupole term, and spin dipole term of the core, are examined. The resulting observational technique is applied to the galaxy 3C66B. This technique is found to under idealized circumstances surpass the technique proposed by Jenet $e t$ al. in accuracy for constraining the mass of SMBHB candidates, but is exceeded in accuracy and precision by Jenet's technique under currently-understood likely conditions. The technique can also under favorable circumstances produce results measurable by currentlyavailable astronomical interferometry such as very-long baseline-interferometry (VLBI).
\end{abstract}

Keywords light deflection $\cdot$ SMBHB $\cdot 3 \mathrm{C} 66 \mathrm{~B} \cdot \mathrm{VLBI}$

\section{Introduction}

The deflection of light by gravity is the oldest experimentally-verified test of the theory of general relativity [1]. With the continued improvement in observational resolution in astronomy, particularly through very-long-baseline interferometry (VLBI), the detection of more subtle effects of this light deflection becomes practical. Consequently, light deflection can be used to measure the properties of distant systems. This paper supplies a theory for using time-variable light deflection to measure or constrain the parameters of binary systems. Specifically, the deflection angle of a light ray from a distant source is related to the configuration

School of Physics and Astronomy, 5, The Parade, Cardiff University, Cardiff, Wales, United Kingdom CF24 3AA.

Tel.: +44(0)2920 875107,

e-mail: Edmund.Schluessel@astro.cf.ac.uk 
and motion of a binary system located in a distant galaxy somewhere between the point of emission of the light ray and its observation.

Super-massive black hole binaries (SMBHBs) are thought to form the cores and primary energy sources of the broad class of galaxies termed "active galaxies", "blazars", or "quasars". However, a combination of distance, radio noise, and optical thickness makes direct observation of presumed SMBHBs impractical. Observing a time-dependent motion in the image of the galaxy can provide information on the mass and orbital parameters of an SMBHB candidate.

Work by Damour and Esposito-Farese [6] and by Kopeikin et al. [5] establishes a theory of time-dependent light deflection by describing the time-

dependent part of the deflection through the quadrupole term, which is the lowestorder term resulting from the mass distrubtion whose effects are practical to evaluate using current astronomical observational techniques. The work of Mashhoon and Kopeikin [25] in examining gravitomagnetic effects furthermore provides a theory for evaluating the contribution of the spin dipole of such systems and complements the work of Einstein [27] in providing a complete theory for stating the location of the deflected image, in the weak field limit. We generalize these theories to a stronger-field regime and put constraints on the theory's applicability in this regime.

As a case study of an active galaxy, the theory is applied to the galaxy $3 \mathrm{C} 66 \mathrm{~B}$, a nearby active galaxy with a candidate SMBHB core [13], and theoretical constraints on 3C66B's parameters from a light deflection experiment are compared to the constraints claimed by Jenet et al. [2].

\section{Theory}

\subsection{Notations, definitions \& assumptions}

We assume that Einstein's theory of general relativity is true to the limits of our ability to observe and applicable to the systems under examination. We do not address MOND or other post-Einsteinian models.

Throughout this paper, "emitter" refers to the source of light rays being observed; "deflector" refers to the mass distribution causing a change in the metric of spacetime from flatness; and "observer" refers to the point where the light rays produced by the emitter are observed.

We also make use of a coordinate system derived from the Cartesian system, defined thus: in a space that is asymptotically Cartesian let a line be described by

$$
x^{i}(t)=k^{i}\left(t-t_{0}\right)+x_{0}^{i} .
$$

Let $t^{*}$ be the time associated with the line's closest approach to the origin of the Cartesian system. Let $\tau=t-t^{*}$ denote a new time coordinate (that is, at $\tau=0$ the line reaches the closest point to the origin of both the Cartesian and projected systems). Space coordinates are projected onto a plane passing through the origin of the coordinate system and perpendicular to a line from the observer to the origin of the coordinate system; these new space coordinates are denoted $\xi^{i}=P^{i j} x^{j}\left(t^{*}\right)$

\footnotetext{
1 Throughout this paper, Greek indices indicate $(0,1,2,3)$ and Latin indices indicate $(1,2,3)$. A contravariant 3 -vector is denoted either in boldface or with a raised Latin index.
} 
where the projection operator is defined $P^{i j} \equiv \delta^{i j}-k^{i} k^{j}$. In the projected coordinate system, the index 0 refers to $\tau$ and the indices $i$ denote coordinates $\xi^{i}$.

For a trajectory described by (1) let $\left.\xi^{j} \equiv P^{i j} x^{i}(\tau)\right|_{\tau=0}$ be the "vector impact parameter" of the trajectory and let $d \equiv\left|\xi^{i}\right|$ be the "scalar impact parameter" of the trajectory. Since the space is asymptotically flat, $d$ is also the ratio of the magnitudes of the angular and linear momenta of the light ray. Note then that for the trajectory described by $x^{i}(\tau), r(\tau)=\sqrt{d^{2}+\tau^{2} 2}$. Let the unit vector $n^{i} \equiv \xi^{i} / d$.

We assume that the wavelengths of all light rays observed are much shorter than the longest wavelength of gravitational radiation emitted by the deflecting system.

\subsection{General theory}

\subsubsection{Background}

Consider a photon emitted at some distant point $x_{0}^{i}$ at some time in the distant past $t_{0}$. This beam of light in asymptotically flat space follows a path $k^{i}$ such that the coordinate $x^{i}$ of the photon is given by the relation (11); therefore, $k^{i}=$ $\left.\left(\partial x^{i} / \partial t\right)\right|_{t=-\infty}$. Let $k^{i}$ be normalized such that $k^{i} k_{i}=1$; then the vector $k^{\alpha}=$ $\left(1, k^{i}\right)$ is parallel to the four-momentum of the photon in flat space.

Let an asymptotically-flat metric $g_{\alpha \beta} \beta^{3}$ be a function of some affine parameter $\lambda$. Let $K^{\alpha} \equiv k^{\alpha}+\kappa^{\alpha}(\lambda)+\Xi^{\alpha}(\lambda)$ be the trajectory of a photon moving in this metric space, where $\kappa^{\alpha}$ describes the part of the trajectory arising from the spherically-symmetric non-flat part of the metric and $\Xi^{\alpha}$ describes the trajectory arising from a perturbation to the metric. Then, we have the geodesic equation [3, equation 87.3]

$$
\frac{d\left(\kappa^{\alpha}+\Xi^{\alpha}\right)}{d \lambda}+\Gamma_{\beta \gamma}^{\alpha} K^{\beta} K^{\gamma}=0 .
$$

The quantity $d\left(\kappa^{\alpha}+\Xi^{\alpha}\right) / d \lambda$ corresponds to the change in momentum of the light ray in space, which when projected onto a plane of observation corresponds to the angular deflection of the light ray. We define this deflection vector by [5,6]

$$
\alpha^{i}\left(t, \xi^{i}\right) \equiv P_{j}^{i}\left[\kappa^{j}+\Xi^{j}\right]_{\text {observer }}+\Delta \alpha^{i},
$$

where the term $\Delta \alpha^{i}$ corresponds to corrections arising from any contribution to deflection other than our deflector.

In the case of Eddington's experiment [1] on solar deflection, the "true" position of the emitter - that is, the position of the emitter observed in the limit of intervening deflection going to zero - was known. In the case of deflectors with small proper motion, in this case extragalactic or otherwise distant objects, where the emitter would be seen without the presence of the intervening deflector may

\footnotetext{
2 Throughout this paper we use the convention $G=c=1$ to simplify our equations.

3 All metrics $g_{\mu \nu}$ in this paper are stated using the harmonic gauge condition, that is, $g^{\mu \nu} \Gamma_{\mu \nu}^{\lambda}=0$. The Minkowski metric in Cartesian coordinates is chosen with signature $(-,+,+,+)$ and is denoted $\eta_{\mu \nu}$, and we make use of the Einstein summation convention.
} 
not be known; therefore, the periastron of the light ray must be determined by other means. Let $P$ be the periastron of the light ray's trajectory about the deflector; in such cases, the time delay between the deflection and the motion of the deflector is related to the periastron by

$$
P=t_{\text {peak deflection }}-t_{\text {alignment }},
$$

where $t_{\text {peak deflection }}$ is the time when the image of the source is observed to be deflected most from the position of the deflector and $t_{\text {alignment }}$ is the time when the projected images of the components of the system and the image fall into a line, assuming that $P<p / 2$ and that the change in the gravitational field propagates at the speed of light.

\subsubsection{Description of deflector}

Our deflector of interest is as follows: two objects are denoted with the indices 1 and 2. The mass of object $1 m_{1} \geq m_{2}$. The objects have positions $x_{1}^{i}(t)$ and $x_{2}^{i}(t)$ and velocities $v_{1}^{i}(t)$ and $v_{1}^{i}(t)$ [FIGURE 1]. Then our source has density distribution

$$
\rho\left(t, x^{i}\right)=m_{1} \delta\left(x^{i}-x_{1}^{i}(t)\right)+m_{2} \delta\left(x^{i}-x_{2}^{i}(t)\right)
$$

and velocity distribution

$$
v^{i}\left(t, x^{j}\right)=v_{1}^{i} \delta\left(x^{j}-x_{1}^{j}\right)+v_{2}^{i} \delta\left(x^{j}-x_{2}^{j}\right)
$$

where $\delta\left(x^{i}\right)$ is the three-dimensional Dirac delta distribution.

Our metric has the form $g_{\mu \nu}=\eta_{\mu \nu}+s_{\mu \nu}+h_{\mu \nu}$ where $s_{\mu \nu}$ is the nonMinkowski part of the Schwarzschild metric and $h_{\mu \nu}$ is a small perturbation. Let $h_{\mu \nu}^{Q}$ be the perturbation resulting from the quadrupole moment of the mass distribution and let $h_{\mu \nu}^{S}$ be the perturbation resulting from the spin dipole of the mass distrubtion. Let the variable $s=t-r$. Then explicitly, the metric is given by [6, 29,25, 26, p 181]:

$$
\begin{aligned}
& s_{00}=\frac{2 m}{r} \\
& s_{0 i}=0 \\
& s_{i j}=\left[\left(1-\frac{2 m}{r}\right)^{-1}-1\right] \frac{x^{i}}{r^{2}} \delta_{i j} ;
\end{aligned}
$$

and

$$
\begin{aligned}
h_{00}^{Q} & =\frac{\partial^{2}}{\partial x^{i} \partial x^{j}} \frac{Q_{i j}(s)}{r}, \\
h_{0 i}^{Q} & =-2 \frac{\partial^{2}}{\partial x^{i} \partial t} \frac{Q_{i j}(s)}{r}, \\
h_{i j}^{Q} & =\frac{\partial^{2}}{\partial x^{i} \partial x^{j}} \frac{Q_{i j}(s)}{r} \delta_{i j}+2 \frac{\partial^{2}}{\partial t^{2}} \frac{Q_{i j}(s)}{r} ;
\end{aligned}
$$


and

$$
\begin{aligned}
& h_{0 i}^{S}=2 \frac{S_{j} x_{k} \epsilon^{i}{ }_{j k}}{r^{3}}, \\
& h_{00}^{S}=h_{i j}^{S}=0,
\end{aligned}
$$

where the vector $S^{i} \equiv\left(J^{23}, J^{31}, J^{12}\right)$ and $J^{i j} \equiv \int\left(x^{i} T^{j 0}-x^{j} T^{i 0}\right) d V$ [26, chapter 2.9].

Let the objects orbit one another with a known period $p$. Let our coordinate system origin be located at the center of mass of the binary and let $m=m_{1}+m_{2}$. Let $a^{i} \equiv x_{1}^{i}-x_{2}^{i}$ be a vector denoting the spatial separation of the two masses and $l \equiv\left|a^{i}\right|$. Let the mass ratio $q \equiv m_{2} / m_{1} \leq 1$.

By our choice of coordinates, the dipole term of the deflector's mass distribution is zero.

Using the Landau-Lifschitz definition of the transverse traceless quadrupole [3, eq 41.3], the quadrupole moment of the deflector is given by:

$$
Q_{i j}(t)=\int \rho(\mathbf{x}, t)\left[3 x_{i} x_{j}-r^{2} \delta_{i j}\right] d V=\frac{m q}{(1+q)^{2}}\left[3 a_{i} a_{j}-l^{2} \delta_{i j}\right] .
$$

In the case that the masses travel in almost circular orbits about their common center of mass, then in a primed coordinate system related to our chosen system only by unitary rotations,

$$
a^{\prime i}(t)=l\left(\begin{array}{c}
\sin \left(\frac{2 \pi t}{p}+\phi^{\prime}\right) \\
0 \\
\cos \left(\frac{2 \pi t}{p}+\phi^{\prime}\right)
\end{array}\right)+\delta a^{\prime i}(t)
$$

where $\phi^{\prime}$ represents a constant phase term, and where $\delta a^{\prime i}$ is small. Rotating from the primed system first about the $y$-axis, then the $x$-axis, then the $z$-axis, we have

$$
a^{i}(t)=l\left(\begin{array}{c}
\cos \Psi \sin \left(\frac{2 \pi t}{p}+\phi\right)+\sin \Psi \sin \Theta \cos \left(\frac{2 \pi t}{p}+\phi\right) \\
-\sin \Psi \sin \left(\frac{2 \pi t}{p}+\phi\right)+\cos \Psi \sin \Theta \cos \left(\frac{2 \pi t}{p}+\phi\right) \\
\cos \Theta \cos \left(\frac{2 \pi t}{p}+\phi\right)
\end{array}\right)+\delta a^{i}(t)
$$

where $\phi$ subsumes rotation about the $y$-axis with $\phi^{\prime}$ and where $\Theta$ and $\Psi$ are the angles of rotation of the plane of motion away from the $x z$-plane about the $x$-and $z$-axes respectively.

\subsubsection{Solution to the geodesic equation}

The theory of the effects of small perturbations to the metric on light propagation in the weak-field limit is already developed [5,6]. However, since the effects of a quadrupolar perturbation fall off as $d^{3}$, it is desirable to expand the theory to be applicable to regions of stronger fields. We note in particular that for a closelyorbiting compact binary system such as a SMBHB, then $m$ and $l$ will be of similar 
magnitude; therefore, we extend the first-order theory of light deflection to order $\mathcal{O}(m / d)^{3}$.

First, note that all terms in (7) are $\mathcal{O}(\mathrm{m} / r)$ or higher and that all terms in (8) are of $\mathcal{O}\left(m l^{2} / r^{3}\right) \leq \mathcal{O}\left(m^{3} / r^{3}\right)$. Let $\mathcal{O}\left(m^{3} / r^{3}\right)$ be small such that all higher orders are negligible. Then, suppressing negligible terms,

$$
\Gamma_{\beta \gamma}^{\alpha}=-\frac{1}{2}\left[\begin{array}{c}
\left(\eta^{\alpha \delta}+s^{\alpha \delta}\right)\left(s_{\beta \delta, \gamma}+s_{\gamma \delta, \beta}-s_{\beta \gamma, \delta}\right) \\
+\left(\eta^{\alpha \delta}\right)\left(h_{\beta \delta, \gamma}+h_{\gamma \delta, \beta}-h_{\beta \gamma, \delta}\right)
\end{array}\right] .
$$

Let the Christoffel symbol associated with the Schwarzschild metric $\Gamma_{\beta \gamma}^{\alpha(\mathbf{S})} \equiv$ $-(1 / 2)\left(\eta^{\alpha \delta}+s^{\alpha \delta}\right)\left(s_{\beta \delta, \gamma}+s_{\gamma \delta, \beta}-s_{\beta \gamma, \delta}\right)$ and the remaining part resulting from the perturbation $\Gamma_{\beta \gamma}^{\alpha(\mathrm{h})} \equiv-(1 / 2)\left(\eta^{\alpha \delta}\right)\left(h_{\beta \delta, \gamma}+h_{\gamma \delta, \beta}-h_{\beta \gamma, \delta}\right)$. Then (2) becomes

$$
\dot{\kappa}^{\alpha}+\dot{\Xi}^{\alpha}+\left(\Gamma_{\beta \gamma}^{\alpha(\mathbf{S})}+\Gamma_{\beta \gamma}^{\alpha(\mathrm{h})}\right)\left(k^{\beta}+\kappa^{\beta}+\Xi^{\beta}\right)\left(k^{\gamma}+\kappa^{\gamma}+\Xi^{\gamma}\right)=0 .
$$

Since all $\Gamma_{\beta \gamma}^{\alpha(\mathrm{S})}$ and all components of $\kappa^{\alpha}$ must be at least of $\mathcal{O}(\mathrm{m} / \mathrm{r})$ or zero, (14) expands, again suppressing negligible terms, to

$$
\dot{\kappa}^{\alpha}+\dot{\Xi}^{\alpha}+\Gamma_{\beta \gamma}^{\alpha(\mathrm{S})}\left(k^{\beta}+\kappa^{\beta}\right)\left(k^{\gamma}+\kappa^{\gamma}\right)+\Gamma_{\beta \gamma}^{\alpha(\mathrm{h})} k^{\beta} k^{\gamma}=0 .
$$

Since

$$
\dot{\kappa}^{\alpha}+\Gamma_{\beta \gamma}^{\alpha(\mathbf{S})}\left(k^{\beta}+\kappa^{\beta}\right)\left(k^{\gamma}+\kappa^{\gamma}\right)=0,
$$

we conclude

$$
\dot{\Xi}^{\alpha}+\Gamma_{\beta \gamma}^{\alpha(\mathrm{h})} k^{\beta} k^{\gamma}=0
$$

which is exactly the result for the weak-field approximation [5,6].

Plugging (16) and (17) into (3) and choosing $\tau$ as our affine parameter, we can define the Schwarzschild $\left(\alpha_{M}^{i}\right)$ and non-Schwarzschild $\left(\alpha_{h}^{i}\right)$ parts of the deflection angle[FIGURE 2]:

$$
\alpha_{M}^{i}\left(\xi^{i}\right) \equiv P_{j}^{i} \kappa^{j}
$$

and

$$
\alpha_{h}^{i}\left(t, \xi^{i}\right) \equiv P_{j}^{i} \Xi^{j}=-\frac{1}{2} P^{i j} \int_{-\infty}^{\infty}\left(h_{\beta \delta, j}+h_{j \delta, \beta}-h_{\beta j, \delta}\right) k^{\beta} k^{\gamma} d \tau .
$$

The monopole term $\alpha_{M}^{i}\left(\xi^{i}\right)$ of the deflection produced by the core is static and unique, regardless of changes of configuration within the core [4]1]. We can use the general, exact solution for $\kappa^{\alpha}$ provided by Darwin [16]:

For purposes of this derivation only, choose spherical coordinates. By the symmetry of the monopole term, this part of the trajectory of the light ray must lie in a plane, so we can choose the coordinate $\theta$ as an affine parameter and the coordinate $\phi$ as constant. Then we obtain an equation of motion

$$
-\frac{r-2 m}{r}\left(\frac{d t}{d \theta}\right)^{2}+\frac{r}{r-2 m}\left(\frac{d r}{d \theta}\right)^{2}+r^{2}=0 .
$$


Identifying the impact parameter with a conserved quantity in the system $\left(r^{3} /(r-2 m)\right)(d t / d \theta)=d$ and substituting in, have three solutions; we discard the two where the light ray never reaches a distant observer and take the remaining one,

$$
\frac{1}{r(\theta)}=-\frac{Q-P+2 m}{4 m P}+\frac{Q-P+6 m}{4 m P} \operatorname{sn}^{2} \zeta(\theta),
$$

where the constant $Q$ is defined by $Q^{2} \equiv(P-2 m)(P+6 m)$, the periastron and impact parameter are related by $d^{2} \equiv P^{3} /(P-2 m)$ and $\zeta(\theta) \equiv \sqrt{(Q / P)}\left(\theta+\theta_{0}\right)$, and $\operatorname{sn} \zeta$ is the Jacobi elliptic sn function [30, 16.1.5]. In the limit of $P \gg m$, inverting this relationship and taking its asymptotic limits at large $r$ leads to the well-known relationship

$$
\alpha_{M, \text { weak field }}^{i}\left(\xi^{i}\right)=\frac{4 m}{d} n^{i}
$$

As $P \rightarrow 3 m$, however, the deflection becomes [28]

$$
\mu\left(\xi^{i}\right)=\ln \frac{m}{d}+\ln [648(7 \sqrt{3}-12)]-\pi \approx \ln \frac{m}{d}+1.248
$$

where $\mu$ is the angle of deflection about the apse of the trajectory, rather than the deflection seen by a distant observer; the angles involved are no longer necessarily small so we cannot approximate $\alpha_{M}=\mu$. In the case of an impact parameter comparable to $3 m$, it is no longer useful to consider the monopolar displacement in and of itself as small differences in impact parameter cause great changes in deflection angle, and multiple images of a source may be detectable, some of which may result from geodesics which travel several times around the deflector. Our consideration therefore must focus not on the static deflection but on timedependent deflections arising from higher multipole moments of the deflector.

Kopeikin and Mashhoon [25] develop the effect of the rotation of a system on that system's deflection of light, in the weak field approximation. Investgation of this effect is useful for the system as described in that every practical case of an astronomical binary will display orbital motion. However, the theory developed by Kopeikin and Mashhoon is only sometimes compatible with the strong-field approximation presented herein.

The integration of (9) is trivial. Let Let $\alpha_{S}^{i}\left(\xi^{i}\right)$ be that part of $\alpha_{h}^{i}$ determined by $h_{\mu \nu}^{S}$. when the deflector is stationary relative to the observer, the resulting contribution is given by

$$
\alpha_{S}^{i}\left(\xi^{i}\right)=\frac{2}{d^{2}}\left(2 S^{j} k^{k} n^{l} \epsilon_{j k l} n^{i}+k^{j} S^{k} \epsilon_{j k}^{i}\right) .
$$

Calculating $S^{i}$ with 12 for the case of a binary whose components are in almost-circular orbits,

$$
S^{i}=-m \frac{q}{1+q} \frac{2 \pi l^{2}}{p}\left(\begin{array}{c}
\frac{q}{(1+q)^{2}-q^{2}(2 \pi l / p)^{2}} \\
+\frac{1}{(1+q)^{2}-(2 \pi l / p)^{2}}
\end{array}\right)\left(\begin{array}{c}
\sin \Psi \cos \Theta \\
\cos \Psi \cos \Theta \\
-\sin \Theta
\end{array}\right)
$$


We must emphasize that (24) is compatible with the $\mathcal{O}\left(\mathrm{m}^{3} / \mathrm{r}^{3}\right)$ generalization above only when $\mathcal{O}\left(m l^{2} / d^{2} p\right) \geq \mathcal{O}\left(m^{3} / d^{3}\right)$; in particular, when $l \approx p$ the system's motion is no longer slow. We draw attention to this contribution to emphasize the difficulty in asssociating an image with a particular source and to underscore the utility of time-dependent deflection versus time-independent deflection in parameterizing a system.

Let $\alpha_{Q}^{i}\left(t, \xi^{i}\right)$ be that part of $\alpha_{h}^{i}$ determined by $h_{\mu \nu}^{Q}$. $\alpha_{Q}^{i}$ is determined by plugging (8) into (19); while [6] uses the method of Fourier transforms, the form of (8) allows direct integration of a Fourier series decomposition as well; either way, the result is the following deflection [5,64:

$$
\alpha_{Q}^{i}\left(t, \xi^{i}\right)=\frac{12}{d^{3}} \frac{m q}{(1+q)^{2}}\left[\left(a_{2}^{2}(s)-a_{1}^{2}(s)\right) n^{i}-a_{1}(s) a_{2}(s) \epsilon_{j k}^{i} k^{j} n^{k}\right]
$$

for which we reiterate the following properties: firstly, in contrast to the monopolar case where $\alpha_{M}^{i}$ always points along $\xi^{i}$, the quadrupolar deflection has a contribution parallel to $\xi^{i}, \alpha_{Q \|}$, and also a contribution perpendicular to $\xi^{i}, \alpha_{Q \perp}$, which vanishes only in the case that a component of the projected quadrupole moment vanishes, that is, only if the axis of rotation of the deflector is perpendicular to our line of sight; and secondly, the deflection depends only on the configuration of the deflector at the time of the light ray's closest approach to the center of mass, $t=t^{*}$.

In the case of almost-circular motion, inserting (12) into (26) leads to

$$
\begin{aligned}
& \alpha_{Q \|}^{i}\left(t, \xi^{i}\right)= \\
& \frac{12 l^{2}}{d^{3}} \frac{m q}{(1+q)^{2}}\left\{\begin{array}{c}
\left.\frac{1}{2} \cos 2 \Psi\left[\begin{array}{c}
\left(1+\sin ^{2} \Theta\right) \cos \left(\frac{4 \pi s}{p}+2 \phi\right) \\
+\sin ^{2} \Theta-1 \\
-\sin 2 \Psi \sin \Theta \sin \left(\frac{4 \pi s}{p}+2 \phi\right)
\end{array}\right]\right\} n^{i}
\end{array}\right.
\end{aligned}
$$

and

$$
\begin{aligned}
& \alpha_{Q \perp}^{i}\left(t, \xi^{i}\right)= \\
& -\frac{6 l^{2}}{d^{3}} \frac{m q}{(1+q)^{2}}\left\{\begin{array}{c}
\frac{1}{2} \sin 2 \Psi\left[\begin{array}{c}
\left(-1+\sin ^{2} \Theta\right) \\
+\left(1+\sin ^{2} \Theta\right) \cos \left(\frac{4 \pi s}{p}+2 \phi\right) \\
+\cos 2 \Psi \sin \Theta \sin \left(\frac{4 \pi s}{p}+2 \phi\right)
\end{array}\right] \epsilon_{j k}^{i} k^{j} n^{k}(28)
\end{array}\right.
\end{aligned}
$$

The relationships (27) and (28) are original to this work and have not previously appeared. From this relationship it is easy to see that the time-dependent deflection of the emitter's image is periodic, with a period half that of the orbit of the core's components.

The greatest time-dependent deflection is observed when the emitter lies on the line of the semimajor axis of the apparent motion; when $q=1$; and when the

\footnotetext{
${ }^{4}$ The symbol $\epsilon_{i j k}$ represents the Levi-Civita permutation symbol defined such that $\epsilon_{123}=1$.
} 
plane of the system lies perpendicular to the plane of observation. In this case, (27) reduces to

$$
\alpha_{Q}(t, d) \leq \frac{3 l^{2}}{2 d^{3}} m\left[\cos \left(\frac{4 \pi s}{p}+2 \phi\right)-1\right]
$$

so the total quadrupolar deflection seen over one half-period of the deflector's motion is

$$
\Delta \alpha_{Q}(d) \leq-\frac{3 l^{2}}{d^{3}} m
$$

Compared to the monopole deflection in the case of a large impact parameter,

$$
\left|\frac{\Delta \alpha_{Q}}{\alpha_{M}}\right| \leq \frac{3 l^{2}}{4 d^{2}}
$$

\subsubsection{Other contributions to the deflection angle}

If the path of the light ray after its closest approach to the deflector but far from the deflector is nearly occulted (for example the Sun or another star), then deflection from this intermediate deflector, $\alpha_{\text {intermediate }}^{i}\left(\xi_{n, \text { int }}^{i}\right)$, must be taken into account as well. Where $\xi_{n, \text { int }}^{i}$ refer to the vector impact parameters of light relative to these intermediate deflectors, $d_{n} \equiv\left|\xi_{n, \text { int }}^{i}\right|$, and $m_{n}$ are the masses of these deflectors, and where $m_{n} / d_{n}$ is small for all $n$,

$$
\alpha_{\text {intermediate }}^{i}\left(\xi_{n}^{i}\right)=-\frac{4 m_{n}}{d_{n}} \frac{\xi_{n, \text { int }}^{i}}{d_{n}} .
$$

In linear approximation and in the harmonic gauge, the various deflections can be superposed linearly. The total deflection of the light ray from our source, therefore, is given by

$$
\alpha^{i}\left(t, \xi^{i}\right)=\alpha_{Q}^{i}\left(t, \xi^{i}\right)+\alpha_{M}^{i}\left(\xi^{i}\right)+\alpha_{S}^{i}\left(\xi^{i}\right)+\alpha_{\text {intermediate }}^{i}\left(\xi_{n, \text { int }}^{i}\right) .
$$

\subsection{Application to 3C66B}

3C66B, also known as 0220+43, is a radio galaxy [9] with $z=0.0215$ [10], approximately $91 \mathrm{Mpc}$ distant from the Milky Way 5 . 3C66B exhibits jets emerging from its core, making it a good candidate for the location of a SMBHB [12].

\footnotetext{
${ }^{5}$ We use a value of $71 \mathrm{~km} / \mathrm{s} / \mathrm{Mpc}$ for the Hubble constant $H_{0}$ for all distance calculations.
} [11] 


\subsubsection{Parameters of the system}

Sudou et al. [13] give estimates of 3C66B's parameters by direct radio observation of its core, including an upper limit on $m$, a period, and an orientation of the core's motion. Sudou also reports a limit on the minimum impact parameter available for determining the parameters of the system using a first-order approximation theory, corresponding to the limit of optical transparency at VLBI's higher operating frequency. The parameters Sudou gives are:

$$
\begin{aligned}
& m \leq 4.4(1+q)^{2} \times 10^{10} \text { solar mass }=6.5(1+q)^{2} \times 10^{15} \mathrm{~cm}, \\
& l \leq 5.1(1+q) \times 10^{16} \mathrm{~cm}, \\
& P \geq 23 \mu \text { as }=3.1 \times 10^{16} \mathrm{~cm}, \\
& d \geq 3.7 \times 10^{16} \mathrm{~cm}, \\
& p=1.05 \pm 0.03 \text { years } \\
& \Theta=15^{\circ} \pm 7^{\circ}
\end{aligned}
$$

where $P$ is constrained by the limit of the core's opacity in the radio spectrum and $\Theta$ is derived from the apparent eccentricities of the elliptical boundaries of radio opacity. From $l$ and $P$ we can furthermore conclude that in the case of maximized $l, q \leq 0.20$.

\subsubsection{Estimates for distant emitters}

Although highly eccentric motion in 3C66B is not ruled out [14], the age of the presumed binary is great enough to have circularized the orbit through gravitational radiation under most conditions [15]. We present the case of circular motion as an upper limit on the time-dependent deflection angle, noting that if all other parameters are constant then in the case of eccentric motion any time-dependent separation of the masses must have $l$ as an upper bound.

Using the maximal figure for mass and the minimal figure for impact parameter in (34) and applying (23), the ratio $m / l=0.30$, placing our proposed system in the regime of strong deflection. We find a monopolar deflection of:

$$
\mu=\ln \left(\frac{6.5 \times 10^{15} \mathrm{~cm}}{3.7 \times 10^{16} \mathrm{~cm}}(1.44)\right)+1.248=0.13 \text { radian }=7.2^{\circ} .
$$

The components of the system as proposed by Sudou have $2 \pi l / p \leq 0.39$. Therefore it is not reasonable to apply (24) to $3 \mathrm{C} 66 \mathrm{~B}$ in the regime where deflection from the quadrupole moment will be detectable.

Deflected images lying along the major axis of the core with the system as constrained in (34) will have time-dependent deflections in the following amounts:

$$
\begin{gathered}
\Delta \alpha_{Q \|}(d) \leq \frac{12 l^{2}}{d^{3}} \frac{m q}{(1+q)^{2}}(1.07), \\
\Delta \alpha_{Q \|}(d) \leq \frac{12\left(5.1 \times 10^{16} \mathrm{~cm}\right)^{2}(1.2)^{2}}{\left(3.7 \times 10^{16} \mathrm{~cm}\right)^{3}}=\frac{\left(6.5 \times 10^{15} \mathrm{~cm}\right)(1.2)^{2}(0.2)}{(1.2)^{2}}(1.07) \\
=5.8 \times 10^{-5} \operatorname{arcsecond}
\end{gathered}
$$


with a period of $p / 2=0.53 \pm 0.02$ yearsfor each component of the deflection.

\section{Observational techniques}

\subsection{Interferometry}

Electromagnetic interferometry provides the best currently-available techniques for high-resolution astronomy. The use of space-based interferometry and improvements in equipment allowing for higher frequencies of observation continue to steadily improve resolution capabilities. The current most powerful technique available is VLBI, which Sudou et al. used to determine the motion in the core of 3C66B [13].

VLBA, the Very Long Baseline Array, is an array of ten radio telescopes [19] operating in wavelengths as short as $3 \mathrm{~mm}$ operating as a single large interferometer. The current best available resolution is

$1.7 \times 10^{-5}$ arcsecond [20], making VLBA currently capable of constraining the parameters of 3C66B further through direct observation as well as the Jenet pulsar timing experiment described below accomplishes indirectly. The launch of the space-based ASTRO-G satellite [21] will extend the resolution capabilities further.

The SIM PlanetQuest mission (formerly Space Interferometry Mission), currently scheduled for launch in 2015 [22], is expected to have a resolution capability of $4 \times 10^{-6}$ arcsecond [23]. SIM will operate in the optical band and quasar observation is part of the planned mission.

Farther into the future, the MAXIM (Micro-Arcsecond X-ray Interferometry Mission) satellite array currently in development [24] is expected to give resolutions on the order of $10^{-7}$ arcsecond in the $\mathrm{x}$-ray band, and is explicitly designed with the observation of black holes in mind.

\subsection{Pulsar timing}

Jenet et al. [2] examined the period of the pulsar PSR B1855+09 for changes in its period over several years, motivated by the idea that as gravitational waves generated by the core of 3C66B pass through the pulsar then the pulsar's signal should be modulated with a period related to the period of the proposed 3C66B SMBHB. The distance between the Solar System and the pulsar furthermore give the advantage that the signals observed modulating the pulsar are some 4000 years older than the motion observed in the 3C66B core, providing some information about the evolution of the system. However, Jenet's experiment produced a null result.

The experiment's analysis involved examining the frequency space of the pulsar's signal for components in a range from $(1 / 27.8) \mathrm{yr}^{-1}$ to $19.5 \mathrm{yr}^{-1}$, then subtracting out the one-year and six-month components resulting from geodetic effects. The results are described as showing no signal distinguishable from noise other than the already-known main oscillation frequencies of the pulsar. Therefore the magnitude of gravitational waves generated by $3 \mathrm{C} 66 \mathrm{~B}$, and consequently the parameters of its core, can be further constrained. 
Jenet et al. assert that a system with $m\left(q /(1+q)^{2}\right)^{3 / 5} \geq 0.7 \times 10^{10}$ solar mass can be ruled out by the observed null result in the change in pulsar periods over seven years; this corresponds in the optimal case of $q=0.2$ to a system with $m=2.3 \times 10^{10}$ solar mass = $3.4 \times 10^{15} \mathrm{~cm}$. For a system under these new constraints, we estimate optimal peak deflections:

$$
\begin{gathered}
\Delta \alpha_{Q \|}(d) \leq \frac{12\left(5.1 \times 10^{16} \mathrm{~cm}\right)^{2}(1.2)^{2}}{\left(3.1 \times 10^{16} \mathrm{~cm}\right)^{3}}\left(3.4 \times 10^{15} \mathrm{~cm}\right) \frac{(0.2)}{(1.2)^{2}}(1.07) \\
=2.1 \times 10^{-5} \operatorname{arcsec} \text { ond } \\
\Delta \alpha_{Q \perp}(d) \leq \frac{12\left(5.1 \times 10^{16} \mathrm{~cm}\right)^{2}(1.2)^{2}}{\left(3.1 \times 10^{16} \mathrm{~cm}\right)^{3}}\left(3.4 \times 10^{15} \mathrm{~cm}\right) \frac{(0.2)}{(1.2)^{2}}(0.26) \\
=5.0 \times 10^{-6} \text { arcsecond }
\end{gathered}
$$

which remains within the detection limit of VLBA as currently configured.

\section{Conclusions}

A theory of light deflection by time-dependent distributions of matter has been presented for metrics which are perturbations of the Schwarzschild metric, accounting for deflection resulting from time-independent and time-dependent terms in the metric. To order $m^{3} / r^{3}$, deflections originating from the quadrupole moment of the mass distribution and, with some constraints, the dipole moment of the system's spin can be linearly superposed on the system as if in a weak-field approximation. The theory can be practically evaluated for and applied to a model of the core of an active galaxy, but the theory of light deflection from the spin of the deflector needs further development for applicability in the regime of strong deflection.

The examination of time-dependent light deflection is a feasible technique for the evaluation of proposed SMBHB systems, under idealized circumstances. In the event that a suitable emitter exists, examination of light deflection can be used to constrain the parameters of the proposed SMBHB in the core of 3C66B. We emphasize that while the existence of an identifiable suitable emitter in the case of 3C66B is unlikely, the theory can be applied equally well to any other SMBHB candidate, any of which may have a suitable source; in particular, active galaxies displaying Einstein rings or other artifacts of strong gravitational lensing, especially multiple images, should be examined. The theory can be equally well applied to intragalactic objects.

The quadrupolar motion in the core of $3 \mathrm{C} 66 \mathrm{~B}$ can be examined and evaluated by the observation of deflected images in the region of the sky near the core of the galaxy, if found; the time-dependent part of the deflection has a magnitude of up to 58 microarcseconds parallel to the impact parameter of the emitter and up to 14 microarcseconds perpendicular to the impact parameter.

For the case of $3 \mathrm{C} 66 \mathrm{~B}$, for most emitters pulsar timing can constrain the parameters of the deflecting system better than time-dependent light deflection can. VLBA in its current configuration is capable of constraining the parameters of the core of $3 \mathrm{C} 66 \mathrm{~B}$ under ideal circumstances. Anticipated interferometers will have 
resolutions up to two orders of magnitude greater and will be capable of evaluating the parameters of the system more closely while examining it in a wide range of frequencies, and may make the observation of time-dependent light deflection resulting from motion in the core of $3 \mathrm{C} 66 \mathrm{~B}$ more practical.

\section{Acknowledgments}

Thanks are given to Prof. Leonid P. Grishchuk of Cardiff University for continued help and support. Prof. Sergei Kopeikin of University of Missouri-Columbia, Dr. Simon Dye of Cardiff University, and Dr. Naomi Ridge formerly of Harvard University provided helpful discussions.

\section{References}

1. Dyson, F.W., Eddington, A.S., Davidson, C.: A Determination of the Deflection of Light by the Sun's Gravitational Field, from Observations Made at the Total Eclipse of May 29, 1919., Philosophical Transactions of the Royal Society of London. Series A, Containing Papers of a Mathematical or Physical Character, 220, 291-333 (1920)

2. Jenet, F.A., Lommen, A., Larson, S.L., Wen, L.: Constraining the Properties of Supermassive Black Hole Systems Using Pulsar Timing: Application to 3C 66B. Ap. J. 606 799-803 (2004)

3. Landau, L.D., Lifshitz, E.M.: The Classical Theory of Fields. Fourth Revised English Edition. Pergamon Press, Oxford (1975)

4. Birkhoff, G.D., Langer, R.: Relativity and Modern Physics. Harvard University Press, Cambridge, Mass., USA (1927)

5. Kopeikin, S.M., Schäfer, G., Gwinn, C.R., Eubanks, M.T.: Astrometric and Timing Effects of Gravitational Waves from Localized Sources. Phys. Rev. D, 59, 084023 (1999); also available as Arxiv preprint gr-qc/9811003 (1998)

6. Damour, T., Esposito-Farese, G.: Light Deflection by Gravitational Waves from Localized Sources. Phys. Rev. D 58, 044003 (1998)

7. Adler, R., Bazin, M., Schiffer, M.: Introduction to General Relativity. Second Edition. McGraw-Hill Inc, New York (1975)

8. Schwarzschild, K.: Über das Gravitationsfeld eines Massenpunktes nach der Einsteinschen Theorie. Sitzungsberichte der Koniglichen Preussischen Akademie der Wissenschaften zu Berlin, Klasse fur Mathematik, Physik, und Technik 189-196 (1916)

9. Northover, K.J.E.: The Radio Galaxy 3C 66, Mon. Not. Roy. Astron. Soc. 165, 369-379 (1973)

10. Matthews, T.A., Morgan, W.W., Schmidt, M.: Ap. J. 140, 35 (1964), quoted in Sudou et al.

11. Spergel, D.N.: et al.: First-Year Wilkinson Microwave Anisotropy Probe (WMAP) Observations: Determination of Cosmological Parameters., Ap. J. Supplement Series 148, 175-194 (2003)

12. Ekejiuba, I.E., Okeke, P.N., Okoye, S.E.: Origin of the Central Radio Gaps in Extragalactic Radio Sources. Ap. \& S. S. 187, 209-214 (1992)

13. Sudou, H., Iguchi, S., Murata, Y., Taniguchi, Y.: Orbital Motion in $3 C$ 66B: Evidence for a Supermassive Black Hole Binary. Science 300, 1263-1265 (2003)

14. De Paolis, F., Ingrosso, G., Nucita, A.A.: A super massive black hole binary in 3C66B: future observational perspectives. A. \& A. 426 379-385 (2004)

15. Croston, J.H., Hardcastle, M.J., Birkinshaw, M., Worrall, D.M.: XMM-Newton observations of the hot-gas atmospheres of $3 C 66 B$ and $3 C 449$. Mon. Not. Roy. Astron. Soc. 4, 10411054 (2003)

16. Darwin, C.G.: The Gravity Field of a Particle. Proceedings of the Royal Society of London, Series A, Mathematical and Physical Sciences 249 180-194 (1959)

17. Giovannini, G., Cotton, W.D., Feretti, L., Lara, L., Venturi, T.: VLBI Obervations of a Complete Sample of Radio Galaxies: 10 Years Later. Ap. J. 552 508-526 (2001) 
18. Fraix-Burnet, D.: An Optical Counterjet in 3C66B?. Mon. Not. Roy. Astron. Soc. 226, 1-7 (1996)

19. New VLBA Site. National Radio Astronomy Observatory (2007) $<$ http://www.vlba.nrao.edu/ $>$

20. Ulvestad, J.: ANGULAR RESOLUTION. National Radio Astronomy Observatory (2006) $<$ http://www.vlba.nrao.edu/astro/obstatus/current/node30.html $>$

21. Hirabayashi, H., et al.: On the Near-term Space VLBI Mission VSOP-2. Arxiv preprint astro-ph/0501020 (2005)

22. MSC-Space Interferometry Mission. Michelson Space Center, California Institute of Technology (2006) <http://msc.caltech.edu/missions/SIMPQ/ $>$

23. Edberg, S.J., Shao, M., Beichman, C.A. ed.: SIM PlanetQuest: A Mission for Astrophysics and Planet Finding. Jet Propulsion Laboratory, California Institute of Technology, Pasadena, California (2005)

24. Gendreau, K.C., et al.: The MAXIM X-ray Interferometry Mission Concept Study. In: From optical to millimetric interferometry: scientific and technological challenges. Proceedings of the 36th Liège International Astrophysics Colloquium, Liège, Belgium, July 2-5, 2001. Surdej, J., Swings, J.P., Caro, D., Detal, A., ed. Université de Liège, Institut d'Astrophysique et de Géophysique, Liège, Belgium 11-16 (2001)

25. Kopeikin, S., Masshoon, B.: Gravitomagnetic Effects in the Propagation of Electromagnetic Waves in Variable Gravitational Fields of Arbitrary-Moving and Spinning Bodies. Phys. Rev. D 65, 064025 (2002)

26. Weinberg, S.: Gravitation and Cosmology. John Wiley \& Sons, Inc., USA (1972)

27. Einstein, A.: Über den Einfluss der Schwerkraft auf die Ausbreitung des Lichtes. In: Annalen der Physik, 35898 (1911). Translated in English in Lorentz, H.A., Einstein, A., Minkowski, H., Weyl, H., The Principle of Relativity. Dover (1952)

28. Bozza, V., Scarpetta, G.: Strong deflection limit of black hole gravitational lensing with arbitrary source distances. Arxiv preprint gr-qc/0705.0246 (2007)

29. Kopeikin, S.M., Makarov, V.: Gravitational bending of light by planetary multipoles and its measurement with microarcsecond astronomical interferometers. Arxiv preprint astro-ph/0611358v2 (2006)

30. Abramowitz, M., Stegun, I.A., ed.: Handbook of Mathematical Functions, ninth printing. National Bureau of Standards Press (1970) 


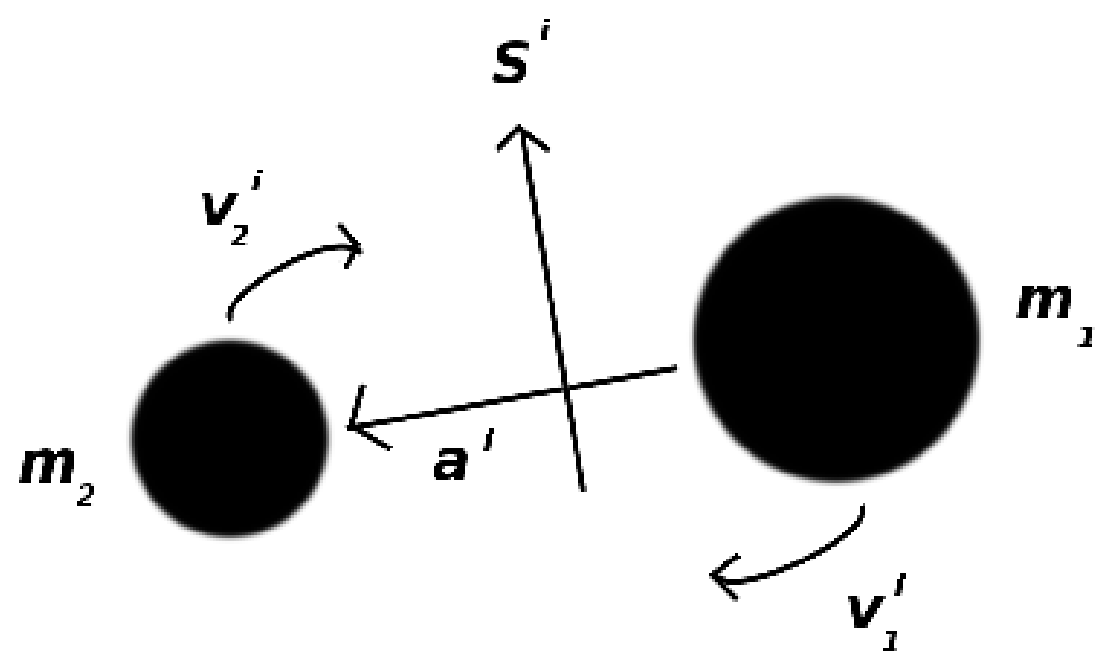

Fig. 1 Object 1 has mass $m_{1}$, velocity $v_{1}^{i}$ and is located as position $x_{1}^{i}$; object 2 has corresponding $m_{2}, v_{2}^{i}$ and $x_{2}^{i} . x_{1}^{i}-x_{2}^{i}=a^{i}$ and the spin vector $S^{i}$ where $S^{i} \equiv\left(J^{23}, J^{31}, J^{12}\right)$ and $J^{i j} \equiv \int\left(x^{i} T^{j 0}-x^{j} T^{i 0}\right) d V$ is perpendicular to $a^{i}, v_{1}^{i}$ and $v_{2}^{i}$.

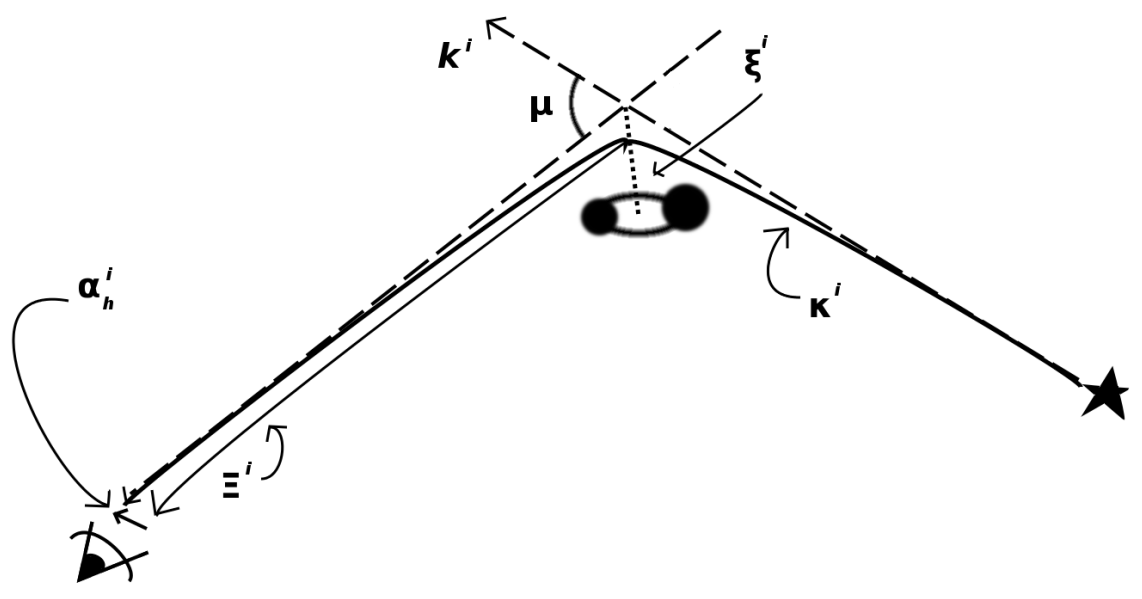

Fig. 2 A light ray produced by the emitter initially follows trajectory $k^{i}$, which has its closest approach to the origin of the coordinate system at $\xi^{i}$. In a pure Schwarzschild space, the light ray follows trajectory $k^{i}+\kappa^{i}(\lambda)$ and is deflected about the apse of its trajectory by angle $\mu$; in a perturbed Schwarzschild space, it follows trajectory $k^{i}+\kappa^{i}(\lambda)+\Xi^{i}(\lambda)$ and the observer records an additional deflection of $\alpha_{h}^{i}$. 
This figure "Figure1.jpg" is available in "jpg" format from: http://arxiv.org/ps/0710.1682v1 
This figure "Figure1.png" is available in "png" format from: http://arxiv.org/ps/0710.1682v1 
This figure "Figure2.jpg" is available in "jpg" format from: http://arxiv.org/ps/0710.1682v1 
This figure "Figure2.png" is available in "png" format from: http://arxiv.org/ps/0710.1682v1 